\title{
Tumor metastático uveal: revisão de literatura sobre a neoplasia ocular maligna mais comum
}

\author{
Uveal metastatic tumor: literature review \\ about the most common eye malignancy
}

Isadora Meyer

\section{RESUMO}

Metástases uveais são consideradas hoje a principal neoplasia maligna ocular. Seu diagnóstico possibilita adoção de medidas que proporcionam redução da tumoração ocular e melhoram a qualidade visual. Diversos tratamentos têm sido propostos para esses fins. Nesse artigo, revisamos as características clínicas, meios de diagnóstico, acompanhamento e padrões nos exames complementares, além dos tratamentos utilizados em pacientes acometidos por essa metástase.

Descritores: Metástase neoplásica; Neoplasias da coróide/secundário; Neoplasias da mama; Neoplasias pulmonares

Médica Residente do Instituto de Olhos do Recife - Recife (PE) - Brasil.

Instituição: Instituto de Olhos do Recife - Recife (PE) - Brasil.

Recebido para publicação em: 19/11/2009 - Aceito para publicação em 20/11/2010 


\section{INTRODUÇÃO}

$\mathbf{U}$ ma proporção significativa de pacientes com neoplasia extraocular (2 a 7\%), principalmen te câncer (CA) de mama e de pulmão, evolui com metástase para o olho. O tumor (TU) metastático uveal é considerado hoje a principal neoplasia maligna ocular, com prevalência maior que o melanoma de coróide, TU maligno ocular primário mais comum em adultos $^{(1-6)}$. Muitos casos não são diagnosticados, por causa da possível apresentação assintomática das lesões e da não realização de exame oftalmológico em pacientes com doença neoplásica avançada ${ }^{(7)}$. Nesse artigo, revisamos as características clínicas, meios de diagnóstico, acompanhamento e padrões dos exames complementares, além dos tratamentos utilizados em pacientes acometidos por metástase uveal, com ênfase para a principal delas, a metástase coroidal.

\section{Quadro clínico}

Os órgãos mais apontados como sítio primário do tumor metastático uveal são pulmão em homens e mama em mulheres ${ }^{(1-5,8-13)}$. Em números absolutos, o tumor de mama aparece como o tumor primário responsável pela maior parte das metástases oculares $(28,6 \%)^{(9)}$. Outros tumores associados à metástase ocular são gastrointestinais, renais, dermatológicos, urogenitais, tireoideanos, de glândula salivar e processos leucêmicos $(1,9,11,14-18)$

A metástase tumoral para a úvea ocorre tipicamente em pacientes entre 40 e 70 anos ${ }^{(7)}$. A disseminação do tumor se dá por via hematogênica e, geralmente, ocorre em coróide (consequente a sua vasta vascularização $)^{(3,4,7,19)}$, seguido por retina e, em raros casos, órbita, disco óptico, íris, pálpebra, corpo ciliar ou vítreo ${ }^{(7,9,17,20,21)}$. O câncer também pode atingir a órbita por infiltração, com deslocamento do globo ocular, compressão do nervo óptico e degeneração paraneoplásica da retina ${ }^{(7,22)}$

Na maioria das vezes (63 a 80,1\%), o acometimento é unilateral ${ }^{(9,23-25)}$. Alguns estudos mostram prevalência maior no olho esquerdo (1,5 para 1$)$ o que pode ser explicado pelo arranjo anatômico do sistema arterial carotídeo. Para atingir a artéria carótida interna direita e, a partir daí, a artéria oftálmica e artérias ciliares do olho direito, os êmbolos tumorais teriam que passar por uma rota mais tortuosa, através da artéria inominada, do que no caminho percorrido pelo lado esquerdo ${ }^{(9)}$.

$\mathrm{O}$ intervalo de tempo entre diagnóstico da neoplasia primária e diagnóstico da lesão ocular é bas- tante variável em diversos estudos ${ }^{(9,24,25)}$. A detecção da metástase pode preceder o próprio diagnóstico do TU primário (principalmente em casos de origem pulmonar ou renal) $)^{(2,7,8,26)}$, ser metástase tardia ou o primeiro sítio de metástase (principalmente no CA de mama $)^{(3,19,25)}$ ou ser a primeira manifestação de recorrência da doença ${ }^{(27,28)}$.A presença de metástases em, pelo menos, outros dois órgãos consiste em fator de risco para a existência de metástase também em coróide $^{(8)}$.

As queixas associadas a essas metástases variam com a localização do tumor intraocular e consistem principalmente em embaçamento ou diminuição da visão ${ }^{(2,7-}$ 9,12,14,21,24,27,29-31) , podendo ocorrer também flashes e floaters ${ }^{(8,9)}$. Em casos de acometimento iriano, com a neovascularização da íris, pode ocorrer aumento da pressão intraocular associado a dor ${ }^{(14,32)}$ ou quadro semelhante a síndrome de efusão uveal ${ }^{(5)}$. Alguns pacientes permanecem assintomáticos ${ }^{(9,24)}$. Pode ocorrer proptose e restrição de movimentos oculares, em casos de extensão extraocular ou de metástase para a órbita associada a metástase para coróide ${ }^{(7,21)}$ (Tabela 1).

As lesões podem ser múltiplas, mas, geralmente, são solítárias, com prevalência de lesões únicas variando de 55,5 a $87,8 \%$ em diversos estudos. Apresentam-se planas (raramente, podem ser pouco elevadas), branco-amareladas ou levemente pigmentadas, associadas a fluido subretiniano e descolamento de retina ${ }^{(1,2,5,8,25)}$. Na maioria das vezes, a localização do(s) tumor(es) é em polo posterior do olho. Da mesma forma que a maior vascularização coroidal favorece maior incidência de metástase, o fato de a área macular apresentar maior vascularização também pode ocasionar maior incidência de metástases em polo posterior do que na periferia. Além disso, há maior proporção de tumores com manifestação clínica nessa localização do que em periferia, leva a maior chance de o diagnóstico não ficar despercebido ${ }^{(7)}$. O diagnóstico diferencial do carcinoma metastático de coróide inclui melanoma de coróide, nevo amelanótico, osteoma de coróide, hemangioma de coróide, neovascularização da coróide com cicatriz disciforme, retinites, coroidites, descolamento retiniano regmatogênico, doença de Harada, síndrome da efusão uveal, coriorretinopatia serosa central e sarcoidose ${ }^{(5-7)}$ (Tabela 2).

\section{Exames diagnósticos e de seguimento}

Os métodos geralmente usados para diagnóstico consistem, inicialmente, em exame oftalmológico completo, incluindo oftalmoscopia binocular indireta, seguido por ecografia, angiografia fluresceínica (AF), angiografia com indocianina verde (AICV) e tomografia 
de coerência óptica (OCT) (Tabela 3). A ressonância nuclear magnética de órbita pode ser usada para avaliar extensão extraocular e afastar a possibilidade de metástase para órbita associada ${ }^{(2)}$. Quando necessário, opta-se por biópsia aspirativa com agulha fina, para confirmação histopatológica do sítio primário. No entanto, na maioria das vezes, a presença de tumor intraocular com características compatíveis com metástase uveal, associada a câncer em outro sítio, confirmado histopatologicamente, conduz ao diagnóstico de metástase uveal, sem necessidade de biópsia do tumor mestastático, mesmo quando esta é a primeira manifestação da doença ${ }^{(9,33)}$.

A AF pode mostrar padrões variáveis, sendo, mesmo assim, útil no diagnóstico diferencial por apresentar bloqueio do contraste e possível detecção de vasos intratumorais. Ao contrário do que ocorre com o hemangioma e com o melanoma, muitos carcinomas metástaticos são hipofluorescentes nas fases arterial e venosa precoces e hiperfluorescentes nas fases subsequentes ${ }^{(7)}$. Entretanto, algumas vezes, tais tumores podem hiperfluorescer precocemente. A AICV apresenta bloqueio da fluoresceína e hipofluorescência no local do tumor ${ }^{(2)}$.

A ecografia é bastante útil, pois ajuda na avaliação de forma, características de refletividade interna e medidas da lesão, sendo ainda mais importante quando existem opacidades dos meios ópticos ou descolamento seroso da retina, que dificultam a fundoscopia ${ }^{(7)}$. A tipo A revela massa com ecogenicidade interna moderada e irregular, e a tipo B, massa sólida com formas inicialmente planas, que podem assumir formas variáveis quando se tornam volumosas ${ }^{(2,29)}$. O OCT possibilita avaliar alterações no epitélio pigmentar de retina (EPR). Revela deslocamento anterior da camada de fotorreceptores por fluido sub-retiniano (espaço hiporrefletivo) e hiperrefletividade do EPR com espessamento do complexo coriocapilar. Depósitos subretinianos de diversos graus de hiperrefletividade podem ser observados, bem como perda da arquitetura normal da retina e separação de suas camadas, identificada por regiões de baixa refletividade. Este exame, assim como os demais de imagem, podem ser utilizados para, além de diagnosticar, observar a evolução da tumoração com o tratamento ${ }^{(5,29,31,34)}$.

\section{Tratamento}

Depende da agressividade, localização e extensão do tumor, dos sintomas, do grau de controle quimioterápico, resposta à radioterapia e do sítio primário, e é realizado pelo oftalmologista, o oncologista e o
Tabela 1

\section{Manifestações clínicas associadas ao TU mestastático de coróide}

Categoria senior
Embaçamento ou diminuição da visão
Flashes ou floaters
Aumento da PIO + dor
Quadro semelhante a síndrome da efusão uveal
Em casos mais avançados
Protose
Restrição dos movimentos oculares

Tabela 2

\section{Diagnósticos diferenciais de TU metastático de coróide}
Melanoma de coróide
Nevus amelanótico
Osteoma de coróide
Hemangioma de coróide
Neovascularização da coróide com cicatriz disciforme
Retinites
Coroidites
Descolamento retiniano regmatogênico
Doença de Harada
Síndrome da efusão uveal
Coriorretinopatia serosa central
Sarcoidose

\section{Tabela 3}

\section{Principais exames complementares na investigação e acompanhamento do TU metastático de coróide}

Oftalmoscopia binocular indireta

Ecografia

Angiografia fluresceínica

Angiografia com indocianina verde

Tomografia de coerência óptica

Ressonância nuclear magnética de órbita

Biópsia aspirativa com agulha fina

radioterapeuta. Alguns tumores metastáticos uveais regridem após o tratamento do tumor primário e não requerem tratamento ocular específico ${ }^{(7)}$. Assim, a conduta é guiada pelo tratamento do tumor primário e pode consistir em simples observação ou incluir quimioterapia, radioterapia, implante de placa de radioterapia, fotocoagulação a laser, terapia hormonal oral (em casos de tumores com receptores hormonais positivos) e intervenções cirúrgicas ${ }^{(1,3,4,14,19,27,35)}$. 
O acompanhamento do paciente deve realizar-se a cada 2 a 4 meses, com exame oftalmológico completo e ecografia ocular para pesquisa de descolamento da retina secundário, o que requer tratamento mais ativo ${ }^{(7)}$.

Os raros casos de metástases de CA de mama para a coróide no sexo masculino apresentam bons resultados com tratamento quimioterápico e com tamoxifeno. CA de mama em homens tem maior concentração de receptores hormonais do que em mulheres, respondendo, portanto, melhor ao tamoxifeno, o que possibilita, muitas vezes, total regressão da lesão ${ }^{(36,37)}$. Há sugestão na literatura de tratamento coadjuvante de CA de mama com metástases para coróide com composto natural derivado de algas marinhas (HESA-A), com melhora da visão e menor necessidade de uso de narcórticos para alívio da dor, sem descrição, no entanto, de diminuição da massa tumoral ${ }^{(38)}$.

Embora, geralmente, ocorra boa resposta do tumor metastático ao tratamento quimioterápico do tumor primário, a radioterapia pode ser usada nos casos em que persiste a baixa acuidade visual por envolvimento macular ou quando, raramente, não há resposta à quimioterapia. Costuma ser efetiva na diminuição do tamanho do tumor, desaparecimento do descolamento retiniano e proliferação do epitélio pigmentar da retina, acompanhados de melhora da acuidade visual ${ }^{(7)}$. No entanto, a radioterapia pode acarretar complicações locais como catarata, olho seco e retinopatia por radiação, entre outras ${ }^{(4)}$. Numa série de 264 pacientes acometidos por metástase uveal de câncer de mama, daqueles submetidos à radioterapia, $5 \%$ desenvolveram catarata; $2 \%$, retinopatia por radiação; $2 \%$, papilopatia por radiação; e $1 \%$, glaucoma neovascular ${ }^{\left({ }^{39}\right)}$. Uma opção que evita essas complicações é a aplicação de laser local. É indicado em casos com limitações para o tratamento sistêmico, como objetivo de melhora da acuidade visual. Assim, a fotocoagulação da lesão com laser diodo pode proporcionar redução do descolamento seroso e da hiperfluorescência à AF, possivelmente pela destruição da vascularização do tumor, causando morte das células cancerígenas. Outra hipótese é a liberação de fatores de modulação extracelular pela ferida induzida pelo laser, o que inclui fatores inibidores da neovascularização do tumor. Ao inibir a angiogênese tumoral, o laser proporcionaria isquemia da massa ${ }^{(4)}$.

Outra opção com indicação semelhante é a termoterapia transpupilar com laser infravermelho. Essa terapia provoca hipertermia do tecido tumoral, com disseminação do calor na área de neovascularização, aquecendo mais essa área do que os tecidos peritumorais.
Proporciona melhora da visão e diminuição do tumor após uma ou duas sessões de laser ${ }^{(1,40,41)}$.

Recentemente, alguns estudos têm sugerido a administração de injeção intravítrea de bevacizumab (Avastin) com regressão parcial do tumor ${ }^{(42)}$. Este mesmo tratamento, com repetidas sessões de injeção intravítrea e em associação com quimioterápico oral (erlotinibe, $150 \mathrm{mg} / \mathrm{dia}$ ) parece proporcionar regressão $\mathrm{da}(\mathrm{s})$ massa(s) e melhora da acuidade visual, sem efeitos colaterais oculares ou sistêmicos. $\mathrm{O}$ bevacizumab combate a angiogênese e o erlotinibe também inibe o crescimento de células tumorais e bloqueia a síntese de proteínas angiogênicas por células tumorais, incluindo o fator de crescimento endotelial vascular ${ }^{(29)}$. Outros quimioterápicos como carboplatina e gencitabina também são descritos com resultados promissores associados ao bevacizumab ${ }^{(43)}$.

Como tentativa de melhora da $\mathrm{AV}$, nos casos em que o tratamento sistêmico se mostra insuficiente ou limitado, também pode ser usada a terapia fotodinâmica com verterporfina, que tem apresentado bons resultados. Assim como, no tratamento proposto para degeneração macular relacionada à idade, injeta-se por via endovenosa a verteporfina (substância fotossensível), que se fixa nos neovasos. Depois de 15 minutos, o produto retido nos neovasos é ativado pelo laser, levando à sua oclusão e isquemia do tumor, sem lesar a parte saudável do tecido ${ }^{(44,45)}$.

A enucleação, exenteração ou excisão cirúrgica do tumor metastático é realizada em casos de crescimento tumoral incontrolável ou de dor por glaucoma secundário associados a importante baixa de visão ${ }^{(7,22)}$.

\section{Prognóstico}

Como em toda neoplasia mestastatizada, o prognóstico do portador desse tumor uveal é preservado. Quando a metástase ocular é a primeira manifestação da doença, obtém-se prognóstico ainda pior, pois isso é indício de neoplasia já bastante disseminada, inclusive para sistema nervoso central ${ }^{(8)}$. A média de sobrevida após o diagnóstico é de 3 a 5 meses nos casos de tumores pulmonares e de 8 a 12 meses nos tumores de mama ${ }^{(7)}$.

\section{Conclusão}

A metástase ocular é rara, porém não é desprezível em portadores de determinadas neoplasias, de forma que sintomas oculares nunca devem ser subestimados nesses pacientes. $\mathrm{O}$ câncer de mama e o de pulmão são os que causam metástases oculares com maior frequência. Com os avanços dos tratamentos oncológicos 
e a possibilidade de cura de neoplasias, a assistência oftalmológica ao doente de câncer mostra-se fundamental, a fim de lhe proporcionar a qualidade de vida que a Oftalmologia se propõe a oferecer, com preservação da visão e conforto ocular.

\section{Abstract}

Choroid metastasis have been considered the most common ocular malignancy. Early detection allows arrangements to provide tumor reduction and to improve visual quality. Many treatments have been proposed to aim these objectives. This work reviews clinical characteristics, diagnosis and monitoring methods, characteristics of complementary exams, and treatments used in patients with this disease.

Keywords: Neoplasm metastasis; Choroid neoplasms/secondary; Breast neoplasms; Lung neoplasms

\section{REFERÊNCIAS}

1. Wang GL, Wang MY, Wei WB. [Clinical features and treatment of choroidal metastasis]. Zhonghua Yan Ke Za Zhi. 2009;45(3):229-33. Chinese.

2. Zhang HR, Ma ZZ, Feng Y, Guo T. [Clinical characteristics of choroidal metastasis]. Zhonghua Yan Ke Za Zhi. 2009;45(4):301-8. Chinese.

3. Nirmala S, Krishnaswamy M, Janaki MG, Kaushik KS. Unilateral solitary choroid metastasis from breast cancer: rewarding results of external radiotherapy. J Cancer Res Ther. 2008;4(4):206-8.

4. Lee SJ, Kim SY, Kim SD. A case of diode laser photocoagulation in the treatment of choroidal metastasis of breast carcinoma. Korean J Ophthalmol. 2008;22(3):187-9.

5. Cury Júnior CE, Cury Júnior A, Siqueira RC, Storti C. Metástase de coróide simulando syndrome de efusão uveal: relato de caso. Arq Bras Oftalmol. 2008;71(2):291-4.

6. Schellini SA, Barros JC, Loureiro VQ, Tagliarini JV, Queiroga E. Melanoma de coróide: relato de caso insuspeito. Rev Bras Oftalmol. 2006;65(1):54-57.

7. Koçak Z, Tabakoðlu E, Benian O, Bayir G, Unlü E, Uzal C. Bilateral choroidal metastases as an initial manifestation of small-cell carcinoma of the lung. Tuberk Toraks. 2006;54(1):61-4.

8. Corrêa ZMS, Burmann TG, Freitas AM, Ramos GZ, Marcon IM. Prevalência de metástases oculares em pacientes com doença metastática reconhecida: resultados preliminares. Arq Bras Oftalmol. 2005;68(2):189-93.

9. Chong JT, Mick A. Choroidal metastasis: case reports and review of the literature. Optometry. 2005;76(5):293-301.

10. Piccinin MRM, Almeida Júnior JA, Aydos RD, Nogueira DC, Silva RF. Metástases de coróide de origem testicular: relato de caso. Arq Bras Oftalmol. 2006;69(6):949-53.

11. Pugnet G, Arista S, Martin-Blondel G, Roche H, Arlet-Suau E, Astudillo L. [Choroidal metastasis as a presenting feature of breast carcinoma]. Rev Med Interne. 2007;28(11):790-2. French.
12. Niu YJ, Liu FL, Zhou ZY, Wang YX, Kang J. [Clinical and pathological analysis of choroidal metastatic carcinoma]. Zhonghua Yan Ke Za Zhi. 2006;42(7):580-4. Chinese.

13. Wang TJ, Yang CM, Ho TC, Huang JS, Lin CP, Yang CH, et al Metastatic choroidal tumors in Taiwan: an 11-year experience. Am J Ophthalmol. 2005;140(4):735-7.

14. Tateno H, Hoshino T, Takahashi K, Matsumura M, Sakaida N, Ohe C. [A rapidly progressed metastatic choroidal tumor from a hepatocellular carcinoma]. Nippon Ganka Gakkai Zasshi. 2009;113(2):107-11. Japanese.

15. Bucerius J, Meyka S, Michael B, Biersack HJ, Eter N. Papillary thyroid carcinoma with an uncommon spread of hematogenous metastases to the choroid and the skin. J Natl Med Assoc. 2008;100(1):104-7.

16. Demirci H, Vine AK, Elner VM. Choroidal metastasis from submandibular salivary gland adenoid cystic carcinoma. Ophthalmic Surg Lasers Imaging. 2008;39(1):57-9.

17. Bianciotto CG, Demirci HY, Shields CL, Shields JA. Simultaneous eyelid and choroidal metastases 36 years after diagnosis of medullary thyroid carcinoma. Ophthal Plast Reconstr Surg. 2008;24(1):62-3.

18. Cormio G, Martino R, Loizzi V, Resta L, Selvaggi L. A rare case of choroidal metastasis presented after conservative management of endometrial cancer. Int $\mathrm{J}$ Gynecol Cancer. 2006;16(6):2044-8.

19. Wickremasinghe S, Dansingani KK, Tranos P, Liyanage S, Jones A, Davey C. Ocular presentations of breast cancer. Acta Ophthalmol Scand. 2007;85(2):133-42. Comment in: Acta Ophthalmol Scand. 2007;85(6):688-9.

20. Benhaddou R, Sayouti A, Khoumiri R, Guelzim H, Gaboune L, Benfdil N, et al. [Iris metastasis from lung cancer]. J Fr Ophtalmol. 2008;31(4):427-9. French.

21. Biswas J, Ho TC, Bhavsar K. Bilateral metastasis to the retina, choroids and optic nerve from breast cancer: a clinicopathological case. Indian J Ophthalmol. 2007;55(1):71-2.

22. Kaur H, Buettner H, Salomao DR, Marks RS. Transcleral orbital invasion by a radiation and chemotherapy-resistant choroidal metastasis of a pulmonary adenocarcinoma. Am J Ophthalmol. 2007;143(2):369-70.

23. Kreusel KM, Bechrakis NE, Wiegel T, Krause L, Foerster $\mathrm{MH}$. Incidence and clinical characteristics of symptomatic choroidal metastasis from lung cancer. Acta Ophthalmol. 2008;86(5):515-9.

24. Soysal HG. Metastatic tumors of the uvea in 38 eyes. Can J Ophthalmol. 2007;42(6):832-5.

25. Kreusel KM, Bechrakis NE, Krause L, Wiegel T, Foerster $\mathrm{MH}$. Incidence and clinical characteristics of symptomatic choroidal metastasis from breast cancer. Acta Ophthalmol Scand. 2007;85(3):298-302.

26. Tazi N, Le Thi Huong D, Bodaghi B, Rixe O, Lehoang P, Piette JC. [Choroidal metastasis revealing pulmonary adenocarcinoma]. Rev Med Interne. 2006;27(9):699-701. French.

27. Jang RW, Doherty M, Hopkins JJ, Warner E. A case of prolonged disease-free survival in a patient with choroidal metastasis from breast cancer. Nat Clin Pract Oncol. 2009;6(2):118-21.

28. Sánchez R, Betancourt L, Sánchez A, Velasquez E, Spinetti D, Cordero C. Choroidal metastasis as first manifestation of systemic recurrence of breast cancer. Breast J. 2008;14(5):498-500.

29. Kim SW, Kim MJ, Huh K, Oh J. Complete regression of choroidal metastasis secondary to non-small-cell lung cancer with intravitreal bevacizumab and oral erlotinib combination therapy. Ophthalmologica. 2009;223(6):411-3. 
30. Asproudis I, Gorezis S, Stefaniotou M, Peschos D, Psilas K. Choroidal metastasis from breast carcinoma. Med Princ Pract. 2006;15(2):153-5.

31. Arevalo JF, Fernandez CF, Garcia RA. Optical coherence tomography characteristics of choroidal metastasis. Ophthalmology. 2005;112(9):1612-9.

32. Kocabora MS, Durmaz S, Bilgic L. Bilateral choroidal metastases presenting as acute glaucoma crisis. Ann Ophthalmol (Skokie). 2008;40(3-4):163-5.

33. Bandyopadhyay S, Adrean SD, Puklin JE, Feng J. Choroidal metastasis from an occult primary diagnosed by fine-needle aspiration: a case report. Diagn Cytopathol. 2009;37(1):38-41.

34. Coffee RE, Jain A, McCannel TA. Ultra wide-field imaging of choroidal metastasis secondary to primary breast cancer. Semin Ophthalmol. 2009;24(1):34-6.

35. El-Saify WM. Choroidal metastasis in a patient with disseminated breast cancer: hormonal therapy-induced regression. Breast J. 2006;12(5):490-2.

36. Shome D, Jayadev C, Gadgil D, Natarajan S, Jain V. Systemic chemotherapy and tamoxifen induced regression of choroidal metastasis from a breast carcinoma in a male. Indian $\mathbf{J}$ Ophthalmol. 2007;55(6):475-7.

37. Cohen VM, Moosavi R, Hungerford JL. Tamoxifen-induced regression of a choroidal metastasis in a man. Arch Ophthalmol. 2005;123(8):1153-4.

38. Ahmadi A, Mohagheghi MA, Fazeli MS, Nahavandian B, Bashardoost N, Musavi Jarahi A, Gharipoor M. HESA-A: new treatment for breast cancer and choroidal metastasis. Med Sci Monit. 2005;11(6):CR300-3.

39. Demirci H, Shields CL, Chao AN, Shields JA. Uveal metastasis from breast cancer in 264 patients. Am J Ophthalmol. 2003;136(2):264-71.
40. Wang TJ, Chen MS, Yang CM, Ho TC. Subthreshold transpupillary thermotherapy for early resolution of foveal subretinal fluid in choroidal metastasis. Retina 2006;26(4):391-5.

41. Pámer Z, Kovács B. [Transpupillary thermotherapy for choroidal metastases]. Magy Onkol. 2005;49(1):59-64. Hungarian.

42. Amselem L, Cervera E, Díaz-Llopis M, Montero J, GarciaPous M, Udaondo P, et al. Intravitreal bevacizumab (Avastin) for choroidal metastasis secondary to breast carcinoma: shortterm follow-up. Eye (Lond). 2007;21(4):566-7.

43. George B, Wirostko WJ, Connor TB, Choong NW. Complete and durable response of choroid metastasis from non-small cell lung cancer with systemic bevacizumab and chemotherapy. J Thorac Oncol. 2009;4(5):661-2.

44. Soucek P, Cihelkova I. Photodynamic therapy with Verteporfin in subfoveal choroidal metastasis of breast carcinoma (a controlled case). Neuro Endocrinol Lett. 2006;27(6):725-8.

45. Isola V, Pece A, Pierro L. Photodynamic therapy with verteporfin of choroidal malignancy from breast cancer. Am J Ophthalmol. 2006;142(5):885-7.
Endereço para correspondência:
Isadora Meyer
Rua Vicente Meira, $n^{\circ} 137$ - Espinheiro
CEP 52020-130 - Recife (PE), Brasil
Tel: (81) 3267-6108 / (81) 8652-7812
Fax: (81) 3423-5353
E-Mail : isadorameyer@yahoo.com.br 